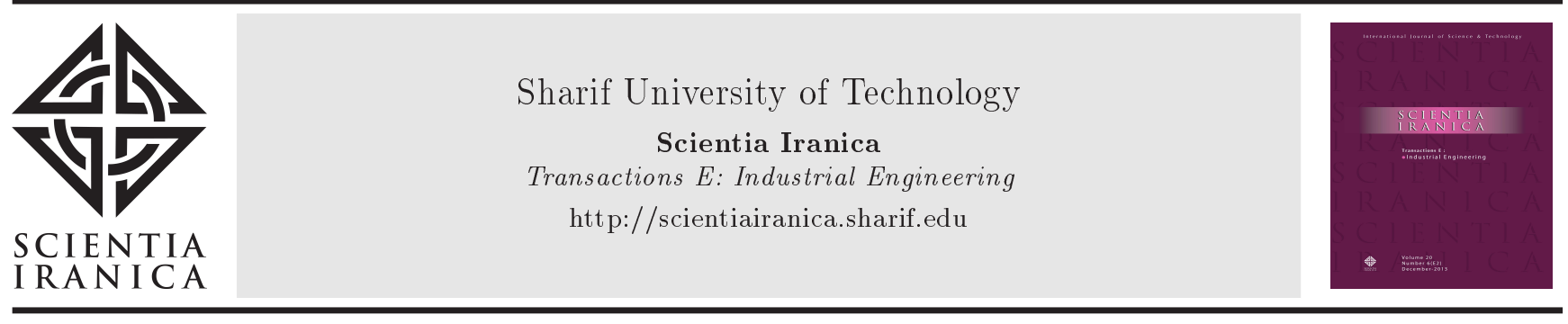

\title{
A novel exact solution algorithm for a robust product portfolio problem under return uncertainty
}

\author{
A. Goli ${ }^{a, 1}$, H. Khademi Zare ${ }^{a, *}$, R. Tavakkoli-Moghaddam ${ }^{b}$, and A. Sadegheih ${ }^{a}$ \\ a. Department of Industrial Engineering, Yazd University, Saffayieh, Yazd, Iran. \\ b. School of Industrial Engineering, College of Engineering, University of Tehran, Tehran, Iran.
}

Received 1 May 2019; received in revised form 13 May 2020; accepted 24 May 2021

\author{
KEYWORDS \\ Robust optimization; \\ Product portfolio \\ selection; \\ Exact solution \\ algorithm; \\ Return; \\ Uncertainty.
}

\begin{abstract}
This research is aimed to address the optimization of a product portfolio problem under uncertainty using the principles of financial portfolios theory. Since the success of a product portfolio is dependent on strategic decision making as well as on future changes of return, the return is best considered when it is deemed an uncertain parameter. The specific innovation of this research is the use of a robust optimization approach and providing an exact solution algorithm based on the model of Bertsimas and Sim. Given the uncertainty of the returns, the product portfolio model was developed based on the robust counterpart formulation of Bertsimas and Sim. An exact solution algorithm was also formulated to reduce the solution time. The results obtained by applying the model to a real case study of the dairy industry in Iran showed that increasing the confidence level would decrease total returns of the portfolio and increase its total risk. A comparison between the proposed algorithm and similar methods showed that, on average, it would make $3 \%$ improvement in the solution time.
\end{abstract}

(C) 2022 Sharif University of Technology. All rights reserved.

\section{Introduction}

In companies that offer multiple products, each product must have a well-defined place in its business strategy. Also, investors often show great interest in the companies' choice of product portfolio, that is, the set of products that the management choose to produce and how they are fitted together to ensure optimal returns over a given time period $[1,2]$.

1. Present address: Department of Industrial Engineering and Future studies, Faculty of Engineering, University of Isfahan, Isfahan, Iran.

*. Corresponding author.

E-mail addresses: Goli.A@eng.ui.ac.ir (A. Goli);

Hkhademiz@yazd.ac.ir (H. Khademi Zare);

tavakoli@ut.ac.ir (R. Tavakkoli-Moghaddam);

Sadegheih@yazd.ac.ir (A. Sadegheih)

doi: $10.24200 /$ sci.2021.53365.3208
Each product has its own characteristics, demand, and profitability, hence requiring specific company resources and raw materials. Accordingly, product portfolios should be designed with careful consideration of all the above details so as to achieve a really high chance of success in securing optimal returns for a company [3]. The science of portfolio management provides the tools and strategies that assist corporate executives and decision-makers to form optimal product portfolios.

Given the extensive scope of discussion on portfolio optimization in the field of economics, Cardoso et al. have recommended that product portfolios are best analyzed using the financial portfolio theory [4]. Accordingly, the present work attempts to use the principles of the financial portfolio theory for the formation of optimal product portfolios. In the financial portfolio theory, the primary objectives of portfolios include both risk minimization and return maximization [3]. 
Therefore, this research also pursues the simultaneous optimization of risk and returns in product portfolio development.

In the remainder of this article, Section 2 reviews the notable studies on the design of product portfolios, Section 3 introduces the proposed mathematical model, Sections 4 presents the robust counterpart model and an algorithm for the exact solution to the model, Section 5 gives the numerical results and their analysis, and finally Section 6 draws conclusions.

\section{Research background}

In a research conducted by Gium et al. [5], a systematic framework was developed in order to improve the efficiency of hospital services by adopting a portfolio approach. They designed optimal portfolios using the Failure Mode and Effect Analysis (FMEA). In another research, Fernandes et al. [6] proposed a new approach for investment in product portfolio with some degree of manufacturing flexibility. Hajnoori et al. [7] presented a two-stage approach to stock portfolio selection based on the Markowitz model. In the first stage, a neural network was employed to predict stock values based on historical data. Then, the Markovitz model was applied in order to form the optimal stock portfolio. Solatikia et al. [8] presented a bi-objective mathematical model for portfolio optimization in fully fuzzy conditions. The objectives of the developed model were to maximize returns and minimize risk formulated as variance in returns. The model was optimized with the help of a crisp weight method. After solving the mathematical model, the results were evaluated and compared with similar findings in the literature.

Takami et al. [9] studied the problem of product portfolio selection in a three-level supply chain where demand for the products was predetermined and multiple products could be handled simultaneously. The objective was to form a product portfolio subject to supply chain constraints such that the chain profit could be maximized. The numerical results of this study showed that adopting a portfolio management approach would have a significant impact on the supply chain profitability. In another research, Esfahani et al. [10] studied the optimization of project portfolios by the Markowitz model. In the mentioned research, two mathematical models were examined. In one model, the objective was to maximize the return of the portfolio by limiting its risk and in the other model, the objective was to minimize the risk of the portfolio by limiting its total revenue to an optimal level. Finally, the two models were combined into a new mathematical model with the objective of maximizing the weighted sum of returns and minimizing the risk of the project portfolio simultaneously. They also developed a harmony search algorithm for solving the mathematical model. The results showed that the proposed algorithm could provide near-optimal solutions to the problem in hand.

Relich and Pawlewski [11] studied the selection of product portfolio under fuzzy conditions. In this research, the importance weights of products were determined by respondents through a questionnaire, expressed by linguistic variables. Then, the linguistic variables were translated into fuzzy numbers. In this work, the objective of product portfolio selection was to achieve the highest fuzzy importance value subject to related production constraints. The numerical results demonstrated the effectiveness of the proposed method and its ability to produce realistic output. Goli et al. [12] proposed a multi-objective mathematical model for product portfolio design. They solved the proposed model with multi-objective invasive weed optimization algorithm. Yevseyeva et al. [13] proposed several portfolio optimization models with limited budget for buying molecules and fixed size of portfolio. Finally, Jiang et al. [14] investigated the performance of NSGAII and SPEA algorithms in optimizing multi-objective portfolio problems. They observed better efficiency of NSGA-II for the problem in hand.

Considering uncertainty can help us find more realistic solutions to the product portfolio problem. In this regard, many researchers have focused on the methods that employ scenario-based approaches, fuzzy numbers, and probabilistic numbers. However, there is still shortcoming in the research on the use of robust optimization approaches in this field. Given the current state of literature, the most important innovation of the present work is offering a robust optimization method for designing product portfolio under uncertainty in product returns.

\section{Product portfolio optimization}

The preliminary goal of any ordinary investment is to gain maximum return with minimum risk. In order to reduce the risk of investment, investors, both natural and legal, prefer to buy a variety of stocks to form a diverse investment portfolio and hence, minimize the effects of periodic fluctuations on their returns. Given the particular importance of risk minimization, investors are always looking for reliable risk assessments to optimize their investment decisions [3]. In the present research, a Markowitz model is developed to optimize product portfolio selection. The developed model is described below:

$$
\begin{aligned}
& \operatorname{Max} \lambda \Sigma_{i=1}^{n} \tilde{\mu}_{i} x_{i}-(1-\lambda) \sum_{i=1}^{n} R_{i} x_{i}, \\
& \sum_{i=1}^{n} u_{i}=k \\
& \sum_{i=1}^{n} x_{i}=1
\end{aligned}
$$




$$
\begin{aligned}
& L B_{i} u_{i} \leq x_{i} \leq U B_{i} u_{i}, \forall i=1, \ldots, n \\
& u_{i} \in\{0,1\}, \forall i=1, \ldots, n \\
& x_{i} \geq 0, \forall i=1, \ldots, n
\end{aligned}
$$

\begin{tabular}{|c|c|}
\hline$i$ & Product index $i=1,2, \ldots, n$ \\
\hline$n$ & Number of products \\
\hline$\tilde{\mu}_{l}$ & Uncertain return of product $i$ \\
\hline$K$ & $\begin{array}{l}\text { Pre-defined number of products to be } \\
\text { placed in the portfolio }\end{array}$ \\
\hline$L B_{i}$ & $\begin{array}{l}\text { Lower bound of investment for product } \\
i \text { if placed in the portfolio }\end{array}$ \\
\hline$U B_{i}$ & $\begin{array}{l}\text { Upper bound of investment for product } \\
i \text { if placed in the portfolio }\end{array}$ \\
\hline$R_{i}$ & Risk of producing product $i$ \\
\hline$\lambda$ & Return priority coefficient \\
\hline$x_{i}$ & $\begin{array}{l}\text { Percentage of investment assigned to } \\
\text { product } i\end{array}$ \\
\hline$u_{i}$ & $\begin{array}{l}\text { Binary variable equal to } 1 \text { if the } \\
\text { investment is done on product } i\end{array}$ \\
\hline
\end{tabular}

where:

Eq. (1) is the objective function of the mathematical model, which maximizes the weighted sum of risk and returns. Eq. (2) states that exactly $K$ products should be selected for inclusion into the product portfolio. Eq. (3) ensures that the percentages of investment in the selected product sum up to $100 \%$. Eq. (4) guarantees that the percentage of investment in any given product remains between predefined minimum and maximum. Eqs. (5) and (6) define the types of decision variables. Since actual returns are assumed to be uncertain, the parameter $\hat{\mu}_{i}$ is used to represent the nominal return for product $i$. This nominal value is assumed to be equal to the mean or expected value of the return for product $i$. The parameter $\sigma_{i}$ represents the standard deviation of the return for product $i$. The parameter $\rho_{i}$ is a coefficient that determines how the actual return fluctuates around its nominal value. In other words, the return of product $i$ fluctuates based on Eq. (7):

$$
\tilde{\mu}_{i} \in\left[\hat{\mu}_{i}-\rho_{i} \sigma_{i}, \hat{\mu}_{i}+\rho_{i} \sigma_{i}\right]
$$

\section{Robust portfolio optimization}

Researchers have proposed several methods, including stochastic programming, feasibility programming, and robust optimization, in order to address the uncertainty in mathematical modeling. Although robust optimization is not a new method, recent advancements made to it have facilitated dealing with uncertainty in optimization problems [8]. In the early steps, Soyster
[15] proposed a robust optimization method, which was too conservative for real-world applications as it tried to produce optimal solutions that would remain feasible even in the worst-case scenarios. Over the past decade, several researchers, including Ben-Tal and Nemirovski [16] and Bertsimas and Sim [17], have proposed less conservative methods with the same purpose. Bertsimas and Sim [17] introduced a non-deterministic model with several innovations in developing the robust counterpart of the linear programming problem (where the linear program was guaranteed to remain linear). They assumed that the returns varied independently in the variation range of the uncertain parameter. They proposed a new term called D-norm, which gained their method many advantages over other robust models.

$$
\begin{aligned}
\|x\|_{d}= & \max _{\{S \cup\{t\}|S \subseteq N,| S|\leq\lfloor d\rfloor, t \in N| S\}} \\
& \left\{\sum_{j \in S}\left|x_{j}\right|+(d-\lfloor d\rfloor)\left|x_{t}\right|\right\}
\end{aligned}
$$

where $x_{j}$ denotes the member of the vector $x, t$ defines the maximization space with the above conditions, and $x_{t}$ is the element of the vector $x$ that meets the specified maximization conditions. As a result, the mathematical model for the portfolio in the present research is modified into $\mathrm{Eq}$. (8) with regard to the Bertsimas robust counterpart formulation.

Maximize $w$

Subject to

$$
\begin{aligned}
& w+(1-\lambda) \sum_{i=1}^{n} R_{i} x_{i}-\lambda\left(\sum_{i=1}^{n} \hat{\mu}_{i} x_{i}-z \Gamma-\sum_{i=1}^{n} \varphi_{i}\right) \\
& \quad \leq 0 \\
& \rho_{i} \sigma_{i} y_{i} \leq z+\varphi_{i}, \quad \forall i=1, \ldots, n, \\
& -y_{i} \leq x_{i} \leq y_{i}, \quad \forall i=1, \ldots, n, \\
& \sum_{i=1}^{n} u_{i}=k, \\
& \sum_{i=1}^{n} x_{i}=1, \\
& L B_{i} u_{i} \leq x_{i} \leq U B_{i} u_{i}, \quad \forall i=1, \ldots, n, \\
& u_{i} \in\{0,1\}, \quad \forall i=1, \ldots, n, \\
& z \geq 0 \quad x_{i} \geq 0 \quad y_{i} \geq 0 \quad \varphi_{i} \geq 0, \quad \forall i=1, \ldots, n,
\end{aligned}
$$

where $\varphi_{i}$ is a variable used for maintaining the convexity of the robust counterpart model [17]. The parameter $\Gamma$ controls the probability of violation of constraints and the objective function getting distanced 
from its ideal value [17]. Bertsimas and Sim [17] call $\Gamma$ the price of robustness. This parameter can take any real value between zero and the number of nondeterministic parameters in the constraint; in other words $\Gamma \in[0, n] . \Gamma=0$ means that all parameters maintain their nominal values and $\Gamma=n$ represents the worst possible state, that is, when all input parameters have uncertainty. In the research conducted by Bertsimas and Sim [17], the upper bound for the probability of violation of constraints that would involve uncertain data was equal to $e^{-\left(\Gamma^{2} / 2 n\right)}$.

\subsection{An exact solution algorithm for the robust portfolio model}

Bertsimas and Pachamanova [18] showed that if a mathematical model had $n$ decision variables and all of the variables had non-deterministic coefficients in the objective function, then instead of solving the robust counterpart model, one could solve $n+1$ modified deterministic models with nominal values. Álvarez et al. [19] have proven that at the robustness level of $\Gamma$, the robust counterpart model of Bertsimas and Sim can be replaced with $n-\Gamma+2$ deterministic models with nominal values for non-deterministic parameters. The exact solution algorithm presented as follows is an expansion of the model proposed by Álvarez et al. [19] for the stock portfolio optimization problem; the proof for the optimality of this algorithm is available in [19]:

Step 1: Sort the products in the decreasing order of $\rho_{i} \sigma_{i}$. In other words, introduce products 1 to $n$ in a way that $\rho_{1} \sigma_{1} \geq \rho_{2} \sigma_{2} \geq \ldots \geq \rho_{n} \sigma_{n}$. Also, consider the product $n+1$ with $\rho_{n+1} \sigma_{n+1}=0$.

Step 2: Determine $\Gamma$ between 0 and $n$.

Step 3: For each $r$ with $r \in\{\Gamma, \Gamma+1, \ldots, n+1\}$, solve the mathematical model presented in Eq. (9). It can be observed that the number of solution rounds for the presented model is equal to $n-\Gamma+2$.

Maximize:

$$
\begin{aligned}
& G_{r}=(1-\lambda) \Sigma_{i=1}^{n} R_{i} x_{i},+\lambda\left(\sum_{i=1}^{n} \hat{u}_{i} x_{i}-\Gamma \rho_{r} \sigma_{r}\right. \\
& \left.-\Sigma_{j=1}^{r}\left(\rho_{j} \sigma_{j}-\rho_{r} \sigma_{r}\right) x_{j}\right), \\
& \sum_{i=1}^{n} u_{i}=k, \\
& \sum_{i=1}^{n} x_{i}=1 \\
& L B_{i} u_{i} \leq x_{i} \leq U B_{i} u_{i}, \quad \forall i=1, \ldots, n, \\
& u_{i} \in\{0,1\}, \quad \forall i=1, \ldots, n, \\
& x_{i} \geq 0, \quad \forall i=1, \ldots, n .
\end{aligned}
$$

Step 4: Determine $r^{*}$ as the best solution found among all the $n-\Gamma+2$ solved problems. In other words, $r^{*}$ can be obtained by Eq. (10):

$$
r^{*}=\arg \max \left\{G_{r} \mid r=\Gamma, \Gamma+1, \ldots n+1\right\} .
$$

Step 5: The optimal value for the objective function as well as the decision variables at the robust level of $\Gamma$ can be obtained by Eq. (11):

$$
W^{*}=G_{r^{*}} \quad x^{*}=x_{r^{*}} \quad u^{*}=u_{r^{*}}
$$

\section{Numerical results}

\subsection{Case study}

The product portfolio optimization model was implemented for Pegah Golpayegan Dairy Company in Iran. This company offers a variety of dairy products in different types and shapes. Naturally, the company can produce multiple dairy products simultaneously. One of the main difficulties ahead of the company is poor decision making in regard to product portfolio formation, which leads to the consumption of significant resources on launching products that fail to yield the expected returns or encounter unacceptable risk of production. Naturally, such products should be removed from the product portfolio of the company [20-21]. From 2012 to 2017 , for example, the company produced 335 different products, but only 52 of these products retained their place in the product portfolio, that is, 283 products were terminated within this period. Of these 283 products, 195 were terminated within their first year of launch, mostly because of decline in sales and high market volatility. Hence, this company is in urgent need of a product portfolio with robustness against changes in returns.

According to the collected information, currently, 85 products can be considered as potential candidates for inclusion in the product portfolio of the company. In this regard, the average profit margin over the past two years was assumed to be a good representative of the nominal return of a product $\left(\hat{\mu}_{i}\right)$. The standard deviation of the returns $\left(\sigma_{i}\right)$ and the parameter $\rho_{i}$ were considered to be $20 \%$.

\subsection{Results of the robust portfolio model}

In order to implement the proposed robust counterpart model, the control parameter $\Gamma$ should be set. For this purpose, first, there should be a clear definition of confidence level under uncertain conditions. In this study, confidence levels of 99\%, 95\%, and 90\% were examined. Then, control parameters were initialized based on the probability of violation in each model. Table 1 shows the values of the control parameter for different confidence levels.

In the portfolio optimization model, the parameter $\lambda$, which determines the degree of preference of returns over risk, can significantly affect the optimal 
Table 1. Values of the control parameter.

\begin{tabular}{cccc}
\hline & \multicolumn{3}{c}{ Bertsimas } \\
\cline { 2 - 4 } & $\mathbf{9 9 \%}$ & $\mathbf{9 5 \%}$ & $\mathbf{9 0 \%}$ \\
\hline$\Gamma$ & 27.75 & 22.55 & 19.77 \\
\hline
\end{tabular}

solution. Since this parameter could take any value between 0 and 1 , the optimal solution was recalculated with $\lambda$ set to $0,0.2,0.4,0.6,0.8$, and 1 .

As per the recommendation of the company, the size of the product portfolio $(K)$ was set to 50 . Using the described setting, the robust counterpart model of Bertsimas was coded in GAMS 14.4 and the globally optimal solution was obtained. Table 2 presents the results of the Bertsimas robust counterpart models with different confidence levels and $\lambda$ values.

In Table 2, the total return was calculated by multiplying the share of each product in portfolio $\left(x_{i}\right)$ by its nominal return $\left(\hat{\mu}_{i}\right)$. Total risk represents the risk of the entire product portfolio based on the optimal values of $x_{i}$, and $W$ is the value of the objective function of the robust counterpart model. As seen in the table, with increase in $\lambda$, the total return and the total risk also increase. This is because a higher $\lambda$ value means that investment will be skewed towards those products that have higher returns, which results in a higher risk too. Therefore, the optimal solutions obtained with different values of $\lambda$ basically indicate the effect of a trade-off between return and risk. Plotting the optimal points obtained for different states of this trade-off results in a curve known as the efficient investment frontier. Figure 1 shows the efficient investment frontier obtained at different confidence levels.

Figure 1 shows that at the efficient investment frontier, as the total return of product portfolio increases, so does its total risk. It can also be seen that as the confidence level increases, the efficient investment frontier moves upward. In other words, at any given level of returns, a higher confidence level

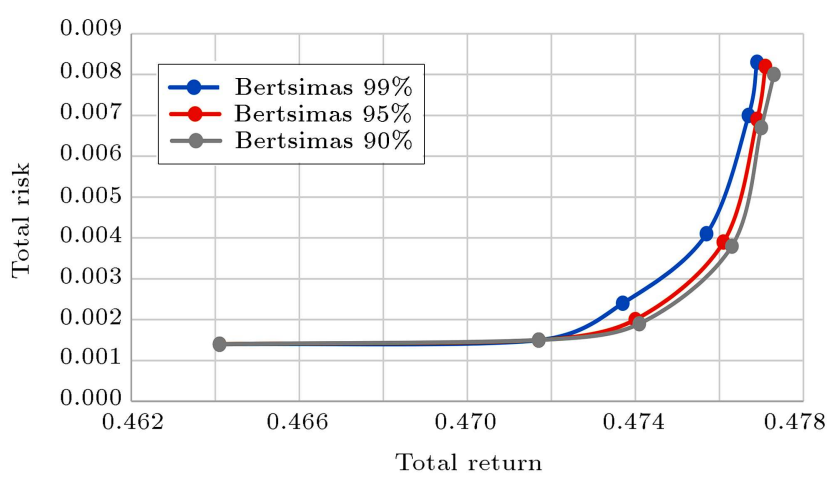

Figure 1. Efficient investment frontier obtained from the Bertsimas robust counterpart model.

corresponds to expecting a higher total risk for the portfolio. This is because a higher confidence level means that the decision made accounts for a greater extent of uncertainty. However, this comes at the expense of a weaker efficient frontier. Accordingly, Figure 1 shows a trade-off between not only risk and return, but also the confidence level and the power of the efficient investment frontier. To better understand this trade-off, in Figure 2, the values of $W$ are plotted against $\lambda$.

In Figure 2, it can be seen that as $\lambda$ increases,

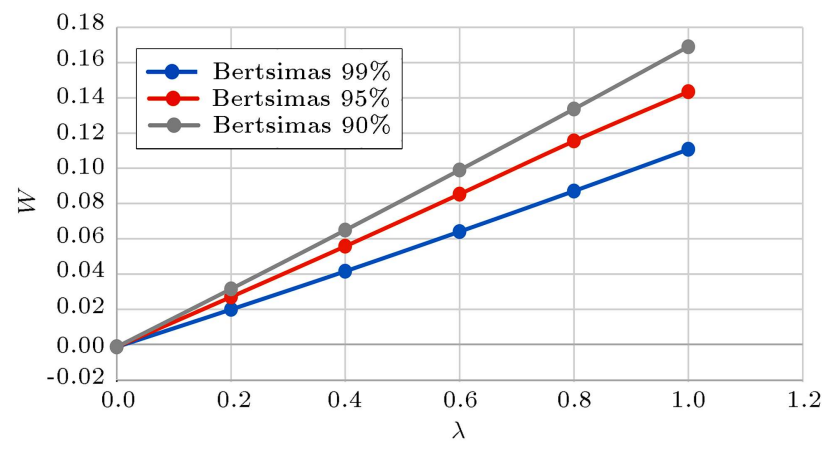

Figure 2. Objective function values for the Bertsimas robust counterpart model at different $\lambda$ values.

Table 2. Results of the Bertsimas robust counterpart model.

\begin{tabular}{cccccccc}
\hline & $\lambda$ & $\mathbf{0}$ & $\mathbf{0 . 2}$ & $\mathbf{0 . 4}$ & $\mathbf{0 . 6}$ & $\mathbf{0 . 8}$ & $\mathbf{1}$ \\
\hline \multirow{3}{*}{ Bertsimas 99\% } & Total return & 0.4641 & 0.4717 & 0.4737 & 0.4757 & 0.4767 & 0.4769 \\
& Total risk & 0.0014 & 0.0015 & 0.0024 & 0.0041 & 0.007 & 0.0083 \\
& $W$ & -0.0014 & 0.0199 & 0.0416 & 0.06413 & 0.08716 & 0.1108 \\
& & & & & & & \\
Bertsimas 95\% & Total return & 0.4641 & 0.4717 & 0.474 & 0.4761 & 0.4769 & 0.4771 \\
& Total risk & 0.0014 & 0.0015 & 0.002 & 0.0039 & 0.0069 & 0.0082 \\
& $W$ & -0.0014 & 0.027 & 0.0558 & 0.0854 & 0.1155 & 0.1435 \\
& & & & & & & \\
Bertsimas 90\% & Total return & 0.4641 & 0.4717 & 0.4741 & 0.4763 & 0.477 & 0.4773 \\
& Total risk & 0.0014 & 0.0015 & 0.0019 & 0.0038 & 0.0067 & 0.008 \\
& $W$ & -0.0014 & 0.0315 & 0.06489 & 0.099 & 0.1336 & 0.169 \\
\hline
\end{tabular}


Table 3. Results of the proposed method for the test examples.

\begin{tabular}{|c|c|c|c|c|c|c|}
\hline \multirow[t]{2}{*}{ \#Example } & \multicolumn{3}{|c|}{ Dimension } & \multicolumn{3}{|c|}{ Solution time } \\
\hline & $N$ & $K$ & $\lambda$ & $\begin{array}{c}\text { Robust counterpart } \\
\text { model }\end{array}$ & $\begin{array}{c}\text { Exact solution } \\
\text { method }\end{array}$ & $\begin{array}{l}\text { Reduction } \\
\text { percentage }\end{array}$ \\
\hline Pr1 & 55 & 20 & 0.3 & 3.451 & 3.441 & $0.29 \%$ \\
\hline $\operatorname{Pr} 2$ & 55 & 20 & 0.7 & 3.419 & 3.416 & $0.08 \%$ \\
\hline $\operatorname{Pr} 3$ & 55 & 50 & 0.3 & 3.296 & 3.285 & $0.33 \%$ \\
\hline $\operatorname{Pr} 4$ & 55 & 50 & 0.7 & 3.297 & 3.275 & $0.67 \%$ \\
\hline $\operatorname{Pr} 5$ & 65 & 20 & 0.3 & 5.776 & 5.556 & $3.81 \%$ \\
\hline $\operatorname{Pr} 6$ & 65 & 20 & 0.7 & 5.792 & 5.674 & $2.04 \%$ \\
\hline $\operatorname{Pr} 7$ & 65 & 50 & 0.3 & 5.599 & 5.476 & $2.20 \%$ \\
\hline $\operatorname{Pr} 8$ & 65 & 50 & 0.7 & 5.612 & 5.474 & $2.45 \%$ \\
\hline $\operatorname{Pr} 9$ & 75 & 20 & 0.3 & 9.572 & 9.309 & $2.75 \%$ \\
\hline $\operatorname{Pr} 10$ & 75 & 20 & 0.7 & 9.488 & 9.205 & $2.98 \%$ \\
\hline $\operatorname{Pr} 11$ & 75 & 50 & 0.3 & 9.125 & 8.794 & $3.62 \%$ \\
\hline $\operatorname{Pr} 12$ & 75 & 50 & 0.7 & 9.137 & 8.636 & $5.49 \%$ \\
\hline $\operatorname{Pr} 13$ & 85 & 20 & 0.3 & 27.443 & 25.944 & $5.46 \%$ \\
\hline $\operatorname{Pr} 14$ & 85 & 20 & 0.7 & 26.094 & 24.773 & $5.06 \%$ \\
\hline $\operatorname{Pr} 15$ & 85 & 50 & 0.3 & 26.171 & 24.842 & $5.08 \%$ \\
\hline $\operatorname{Pr} 16$ & 85 & 50 & 0.7 & 26.169 & 24.379 & $6.84 \%$ \\
\hline \multicolumn{4}{|c|}{ Average } & 11.2151 & 10.7174 & $3.07 \%$ \\
\hline
\end{tabular}

the value of the objective function $(W)$ increases too. This is because of higher variation in returns and lower variation in risk as $\lambda$ increases. For example, in the Bertsimas model 99\%, raising $\lambda$ from 0 to 1 increases the total return by about 0.01 , but increases the total risk by only 0.007. Hence, the effect of increased returns easily surpasses the effect of increased risk, giving $W$ an ascending trend. This figure also shows that increasing the confidence level from $90 \%$ to $99 \%$ decreases the value of $W$ at all levels of $\lambda$. This means that, since the objective is to maximize $W$, increasing the confidence level translates into a lower expected quality of the portfolio. This difference is very small at $\lambda=0$, but increases as we move toward $\lambda=1$. Therefore, it can be concluded that there is always a trade-off among the level of confidence in solutions, the preference of decision-makers for returns versus risk, and the expected quality of the portfolio; a tradeoff that every decision-maker has to consider in their analysis.

\subsection{Efficiency of the proposed Bertsimas solution method}

In order to evaluate the efficiency of the proposed algorithm, 16 examples with different dimensions were constructed using the case study data. The examples were matched in the Bertsimas model and optimized using GAMS software. The solution times were recorded. Then, the algorithm introduced in Section 4.1 was implemented in the same software and the solution time recorded. A summary of the results is presented in Table 3.

As shown in Table 3, the exact solution algorithm was able to reduce the solution time between $0.08 \%$ and $6.84 \%$. However, with increasing dimensions, the solution time significantly increased and with increasing problem dimensions, the proposed algorithm performed better and reduced the solution time at higher percentages. Figure 3 compares solution times for the two methods.

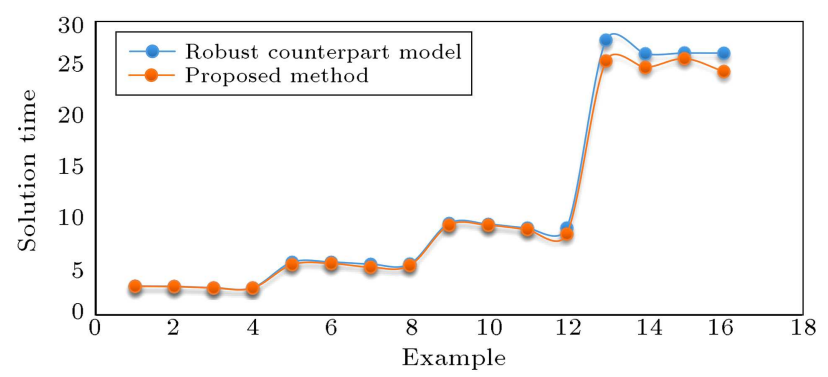

Figure 3. Comparison of the solution times of the two methods. 
As shown in Figure 3, with increase in the dimensions of an examples, the solution time increased sharply, hence the greatest time savings were achieved with examples 14-16. Because the choice of the product portfolio is a strategic decision, the exact solution method should be used in large dimensions. However, the robust counterpart model takes a too high solution time by increasing the dimensions of the problem. Therefore, it can be concluded that the proposed method is a very suitable means for solving the robust product portfolio problem in large dimensions.

\section{Conclusion and directions for future research}

In this research, a robust optimization for the product portfolio problem was presented. The goal was to reduce the risk of investment and increase the return on investment in various products. Given that the portfolio selection problem was a strategic decision and an exact solution could be achievable in a short or reasonable time, the numerical results obtained from the exact solution were analyzed.

Since the purpose of the optimization model was simultaneous consideration of risk and return, and the level of confidence was also adjustable in the robust counterpart model, the final output of each model was an investment efficiency boundary under different levels of confidence. The analysis carried out in this research would help decision-makers choose the most appropriate solution among the possible ones depending on the circumstances. In order to further develop the present research, one can consider the uncertainty in the return for products to be gray as well as fuzzy and compare the results with the robust optimization approaches.

\section{References}

1. Jiao, J. and Zhang, Y. "Product portfolio planning with customer-engineering interaction", IIE Transactions, 37(9), pp. 801-814 (2005).

2. Bazmohammadi, S., Foroud, A.A., and Bazmohammadi, N. "Portfolio optimization in electricity market using a novel risk based decision making approach", Scientia Iranica, Transactions D, 25(6), pp. 3569-3583 (2018).

3. Biglova, A., Ortobelli, S., Rachev, S.T., et al. "Different approaches to risk estimation in portfolio theory", The Journal of Portfolio Management, 31(1), pp. 103112 (2004).

4. Cardozo, R.N. and Smith Jr, D.K. "Applying financial portfolio theory to product portfolio decisions: An empirical study", The Journal of Marketing, 47(2), pp. 110-119 (1983).
5. Geum, Y., Shin, J., and Park, Y. "FMEA-based portfolio approach to service productivity improvement", The Service Industries Journal, 31(11), pp. 1825-1847 (2011).

6. Fernandes, R., Gouveia, J.B., and Pinho, C. "Product mix strategy and manufacturing flexibility", Journal of Manufacturing Systems, 31(3), pp. 301-311 (2012).

7. Hajnoori, A., Amiri, M., and Alimi, A. "Forecasting stock price using grey-fuzzy technique and portfolio optimization by invasive weed optimization algorithm", Decision Science Letters, 2(3), pp. 175-184 (2013).

8. Solatikia, F., Kiliç, E., and Weber, G.W. "Fuzzy optimization for portfolio selection based on embedding theorem in fuzzy normed linear spaces", Organizacija, 47(2), pp. 90-97 (2014).

9. Takami, M.A., Sheikh, R., and Sana, S.S. "Product portfolio optimisation using teaching-learning-based optimisation algorithm: a new approach in supply chain management", International Journal of Systems Science: Operations \& Logistics, 3(4), pp. 236-246 (2015).

10. Esfahani, H.N., Sobhiyah, M.H., and Yousefi, V.R. "Project portfolio selection via harmony search algorithm and modern portfolio theory", Procedia - Social and Behavioral Sciences, 1(226), pp. 51-58 (2016).

11. Relich, M. and Pawlewski, P. "A fuzzy weighted average approach for selecting portfolio of new product development projects", Neurocomputing, 1(231), pp. 19-27 (2017).

12. Goli, A., Zare, H.K., Tavakkoli-Moghaddam, R., et al. "Application of robust optimization for a product portfolio problem using an invasive weed optimization algorithm", Numerical Algebra, Control \& Optimization, 9(2), pp. 187-209 (2019).

13. Yevseyeva, I., Lenselink, E.B., de Vries, A., et al. "Application of portfolio optimization to drug discovery", Information Sciences, 1(475), pp. 29-43 (2019).

14. Jiang, Z., Wang, H., Zhang, H., et al. "Value recovery options portfolio optimization for remanufacturing end of life product", Journal of Cleaner Production, 210, pp. 419-431 (2019).

15. Soyster, A.L. "Convex programming with set-inclusive constraints and applications to inexact linear programming", Operations Research, 21(5), pp. 1154-1157 (1973).

16. Ben-Tal, A. and Nemirovski, A. "Selected topics in robust convex optimization", Mathematical Programming, 112(1), pp. 125-158 (2008).

17. Bertsimas, D. and Sim, M. "The price of robustness", Operations Research, 52(1), pp. 35-53 (2004).

18. Bertsimas, D. and Pachamanova, D. "Robust multiperiod portfolio management in the presence of transaction costs", Computers \& Operations Research, 35(1), pp. 3-17 (2008). 
19. Álvarez-Miranda, E., Ljubić, I., and Toth, P. "A note on the Bertsimas \& Sim algorithm for robust combinatorial optimization problems", $4 O R, \mathbf{1 1}(4)$, pp. 349-360 (2013).

20. Goli, A., Babaee Tirkolaee, E., and Soltani, M. "A robust just-in-time flow shop scheduling problem with outsourcing option on subcontractors", Production \& Manufacturing Research, 7(1), pp. 294-315 (2019).

21. Goli, A., Zare, H.K., Tavakkoli-Moghaddam, R., et al. "Multiobjective fuzzy mathematical model for a financially constrained closed-loop supply chain with labor employment", Computational Intelligence, 36(1), pp. 4-34 (2020).

\section{Biographies}

Alireza Goli was born in Isfahan, Iran, in 1989. He received his BSc and MSc degrees in Industrial Engineering from Golpayegan University of Technology, Iran, in 2013 and Isfahan University of Technology, Iran, in 2015, respectively. Then, he received a $\mathrm{PhD}$ degree in Industrial Engineering from Yazd University, Iran, in 2019. Now, he is a lecturer at Isfahan University, which is one of the famous universities in Iran. He has published more than 60 papers in high-quality journals and conferences and has been serving as a reviewer in many reputed journals such as IEEE Transactions on Fuzzy Systems, the Journal of Supercomputing, and the Annals of Operations Research. He was an excellent reviewer winner of Publons in 2019. He is also working as a member of the editorial board in different journals like the Journal of Applied Research in Industrial Engineering and the International Journal of Applied Optimization Studies. His current research interests include supply chain management, disaster relief optimization, meta-heuristic algorithms, robust optimization, artificial intelligence, and portfolio management.

Hasan Khademi Zare is a Professor of Industrial Engineering at the College of Engineering, Yazd University, Iran. He obtained his $\mathrm{PhD}$ in Industrial Engineering from Amirkabir University of Technology in Iran. Professor Hassan Khademi Zare has published three books and more than 500 papers in reputable academic journals and conferences.

Reza Tavakkoli-Moghaddam is a Professor of Industrial Engineering at the College of Engineering, University of Tehran, Iran. He obtained his PhD in Industrial Engineering from Swinburne University of Technology in Melbourne in 1998, his MSc in Industrial Engineering from the University of Melbourne in Melbourne in 1994, and his BSc in Industrial Engineering from the Iran University of Science and Technology in Tehran in 1989. He is the recipient of the 2009 and 2011 Distinguished Researcher Awards and the 2010 and 2014 Distinguished Applied Research Awards at University of Tehran, Iran. He has been selected as a National Iranian Distinguished Researcher in 2008 and 2010. Professor Tavakkoli-Moghaddam has published four books, 17 book chapters, and more than 790 papers in reputable academic journals and conferences. Ahmad Sadegheih is a Professor of Industrial Engi-
neering at the College of Engineering, Yazd University,
Iran. He obtained his PhD in Industrial Engineering
from Cardiff University in England. Professor Ahmad
Sadegheih has published two books and more than 300
papers in reputable academic journals and conferences. 